\title{
Gallium-67 SPECT-CT for the evaluation of head and neck: preliminary study on maximum standardised uptake value in lesions, and in the parotid and submandibular glands
}

\author{
Makoto Oohashi ${ }^{1 B, F}$, Hiroo Toshima ${ }^{2 C, F}$, Kazuhide Hayama ${ }^{20}$,Ichiro Ogura ${ }^{2 A, E, G}$ \\ 'Dental Anaesthesia and General Health Management, The Nippon Dental University Niigata Hospital, Niigata, Japan \\ 2Department of Oral and Maxillofacial Radiology, The Nippon Dental University School of Life Dentistry at Niigata, Niigata, Japan
}

\section{Abstract}

Purpose: The aim of this study was to investigate the gallium-67 $\left({ }^{67} \mathrm{Ga}\right)$ single-photon emission-computed tomography (SPECT-CT) for the evaluation of head and neck, especially maximum standardised uptake value (SUV ${ }_{\text {max }}$ ) in lesions, and in the parotid and submandibular glands.

Material and methods: Fourteen patients with head and neck lesions who underwent SPECT-CT at 72 hours after injection of ${ }^{67} \mathrm{Ga}$ were included in this study. The ${ }^{67} \mathrm{Ga}$ SPECT-CT parameters SUV ${ }_{\text {max }}$ were compared between the parotid glands and submandibular glands. Statistical analyses for the SUV $\mathrm{max}_{\max }$ were performed by Mann-Whitney $U$ test. A $p$ value lower than 0.05 was considered as statistically significant.

Results: Of all 14 patients, ${ }^{67} \mathrm{Ga}$ SPECT-CT was positive for two cases (one malignant lymphoma and one osteomyelitis). The $\mathrm{SUV}_{\max }$ for malignant lymphoma and osteomyelitis were 11.21 and 10.95, respectively. Furthermore, $\mathrm{SUV}_{\max }$ for parotid glands and submandibular glands of all patients were $4.86 \pm 1.89$ and $4.64 \pm 1.64$, respectively $(p=0.694)$.

Conclusions: This study investigated the ${ }^{67} \mathrm{Ga}$ SPECT-CT for the evaluation of head and neck, especially SUV $\mathrm{S}_{\max }$ of lesions, and the parotid and submandibular glands. ${ }^{67} \mathrm{Ga}$ SPECT-CT may be an effective technique for the evaluation of maxillofacial lesions.

Key words: gallium radioisotopes, SPECT/CT, carcinoma, inflammation, salivary gland.

\section{Introduction}

Gallium-67 $\left({ }^{67} \mathrm{Ga}\right)$ scintigraphy is a useful adjunct tool for differentiation of malignant tumours from benign tumours or inflammatory disease in the oral and maxillofacial region [1-4]. ${ }^{67} \mathrm{Ga}$ scintigraphy is an effective technique for the evaluation of head and neck squamous cell carcinoma (SCC), especially tumour recurrence and distant metastases [5]. Furthermore, ${ }^{67} \mathrm{Ga}$ single-photon emission tomography (SPECT) substantially increases confidence in the diagnosis of head and neck tumours when computed tomography (CT) and/or magnetic resonance imaging
(MRI) do not permit differentiation between benign and malignant disease [6]. With the exception of SCC, some authors have reported that ${ }^{67} \mathrm{Ga}$ scintigraphy is useful in the differentiation of malignant lymphoma [7], sarcoidosis [8-10], and other inflammatory diseases $[11,12]$.

In recent years, SPECT-CT scanners provide fusion images of CT and SPECT and also produce attenuation correction maps that are necessary for quantitative analyses using the standardised uptake value (SUV) [13]. SUV is defined as the tissue concentration of tracer as measured by a scanner divided by the activity injected divided usually by body weight [14]. Quantitative salivary

Correspondence address:

Ichiro Ogura, Department of Oral and Maxillofacial Radiology, The Nippon Dental University School of Life Dentistry at Niigata, 1-8 Hamaura-cho, Chuo-ku,

Niigata, Niigata 951-8580, Japan, phone: +81-25-267-1500, fax: +81-25-267-1134, e-mail: ogura@ngt.ndu.ac.jp

Authors' contribution:

A Study design · B Data collection · C Statistical analysis · D Data interpretation - E Manuscript preparation · F Literature search · G Funds collection 
gland SPECT-CT holds promise as an objective imaging modality for assessment of salivary dysfunction $[15,16]$. Furthermore, SUV derived from bone SPECT-CT could be useful for the evaluation of osteonecrosis of the jaw $[17,18]$. However, to the best of our knowledge, ${ }^{67} \mathrm{Ga}$ SPECT-CT for the evaluation of head and neck have not been reported in the literature. The aim of this study was to investigate the ${ }^{67} \mathrm{Ga}$ SPECT-CT for the evaluation of head and neck, especially maximum standardised uptake value $\left(\mathrm{SUV}_{\max }\right)$ in lesions, and in the parotid and submandibular glands.

\section{Material and methods}

\section{Patients}

The Ethics Committee of our institution approved this retrospective study. After providing written informed consent, 14 patients (six male, eight female; range age 25-83 years, mean age 65.1 years) underwent ${ }^{67} \mathrm{Ga}$ SPECT-CT at our university hospital from November 2018 to September 2019. The histopathological diagnoses were obtained by surgery or biopsy in all cases (three verrucous carcinoma [two tongue and one mandible], three epithelial dysplasia [one tongue, one buccal mucosa, and one floor of the mouth], one squamous cell carcinoma [tongue], one malignant lymphoma [neck], one osteoradionecrosis [mandible], one osteomyelitis [mandible], and four postoperative follow-up of malignant tumours [palatal adenoid cystic carcinoma, palatal squamous cell carcinoma, mandibular squamous cell carcinoma, and maxillary malignant melanoma]).

\section{Image acquisition}

SPECT/CT was obtained with an Optima NM/CT 640 (GE Healthcare Japan, Tokyo, Japan) at 72 hours after the injection. The radiopharmaceutical used in this study was ${ }^{67} \mathrm{Ga}$-citrate $\left({ }^{67} \mathrm{Ga}\right.$-citrate NMP, Nihon Medi-Physics, Tokyo, Japan). Each patient was administered the agent at $111 \mathrm{MBq}$ with a rapid intravenous injection. The SPECT scans were acquired using medium-energy general-purpose collimation with 93, 184, and $300 \mathrm{keV}$ photoenergy peaks for ${ }^{67} \mathrm{Ga}$, a $128 \times 128$ matrix of $4.2 \mathrm{~mm}$ pixel size, and a total of 60 projections (30 stops) over $360^{\circ}$ with a dwell time of $20 \mathrm{~s} / \mathrm{stop}$. Subsequent to the SPECT acquisition, a low-dose CT scan was acquired with $120 \mathrm{kV}$ and $20 \mathrm{~mA}$ using a $512 \times 512$ matrix. The CT data were generated with a $2.5 \mathrm{~mm}$ slice thickness.

\section{Image analysis}

To calculate the SUV, the SPECT/CT system was first calibrated to the dose calibrator for determination of the system sensitivity and the converting factor for radioactivity from measured counts. SUV in a given volume of interest (VOI) was indirectly calculated from the percentage of injected dose, which was obtained by using dosimetry software (Q. Metrix; GE Healthcare Japan). To derive the percentage of injected dose in a certain VOI by using dosimetry software, we entered the following information into the software in advance: the pre-injection radioactivity in the syringe and the measurement time, the post-injection residual radioactivity in the syringe and the measurement time, the time of injection to the patient, body weight, and the system sensitivity. On a dedicated workstation (GEniE Xeleris; GE Healthcare Japan), CT, SPECT, and SPECT/CT images were displayed with the dosimetry software. By using the transaxial and coronal CT images as the anatomic reference, a VOI was drawn over the lesions, the bilateral parotid gland, and the submandibular gland, which was automatically reflected on the SPECT/CT fusion images. Then the dosimetry software provided multiple quantitative data for a given VOI. The maximum SUV ( $\left.\mathrm{SUV}_{\max }\right)$ in a given VOI was calculated as follows: $\mathrm{SUV}_{\max }=$ (maximum radioactivity/voxel volume)/(injected radioactivity/body weight).

\section{Statistical analysis}

The ${ }^{67} \mathrm{Ga}$ SPECT-CT parameters of $\mathrm{SUV}_{\max }$ were compared between the parotid glands and submandibular glands. Statistical analyses for the $\mathrm{SUV}_{\max }$ were performed by Mann-Whitney $U$ test. These analyses were performed with the statistical package IBM SPSS Statistics, version 26 (IBM Japan, Tokyo, Japan). A $p$ value lower than 0.05 was considered as statistically significant.

\section{Results}

Of all 14 patients, ${ }^{67} \mathrm{Ga}$ SPECT-CT was positive for two (one malignant lymphoma [Figures 1 and 2] and one osteomyelitis [Figures 3 and 4]). The SUV ${ }_{\max }$ for malignant lymphoma and osteomyelitis were 11.21 and 10.95, respectively. However, the other 12 patients were negative.

Table 1 shows 14 cases of ${ }^{67}$ Ga SPECT-CT SUV max $_{\text {of }}$ of parotid and submandibular glands. $\mathrm{SUV}_{\max }$ for parotid glands and submandibular glands of all patients were 4.86 \pm 1.89 and $4.64 \pm 1.64$, respectively $(p=0.694)$. Furthermore, there was no significant relationship between age $(p=0.544)$, gender $(p=0.320)$, and $\mathrm{SUV}_{\max }$ for parotid glands and submandibular glands.

\section{Discussion}

${ }^{67} \mathrm{Ga}$ scintigraphy has been widely used to detect various malignant neoplasms, such as SCC [1,6] and malignant lymphoma [7] of the head and neck. In our study, ${ }^{67} \mathrm{Ga}$ SPECT-CT for two of 14 patients with head and neck lesions were positive (one malignant lymphoma and one osteomyelitis). However, the other 12 patients were negative. These 12 histopathological diagnoses were three verrucous 

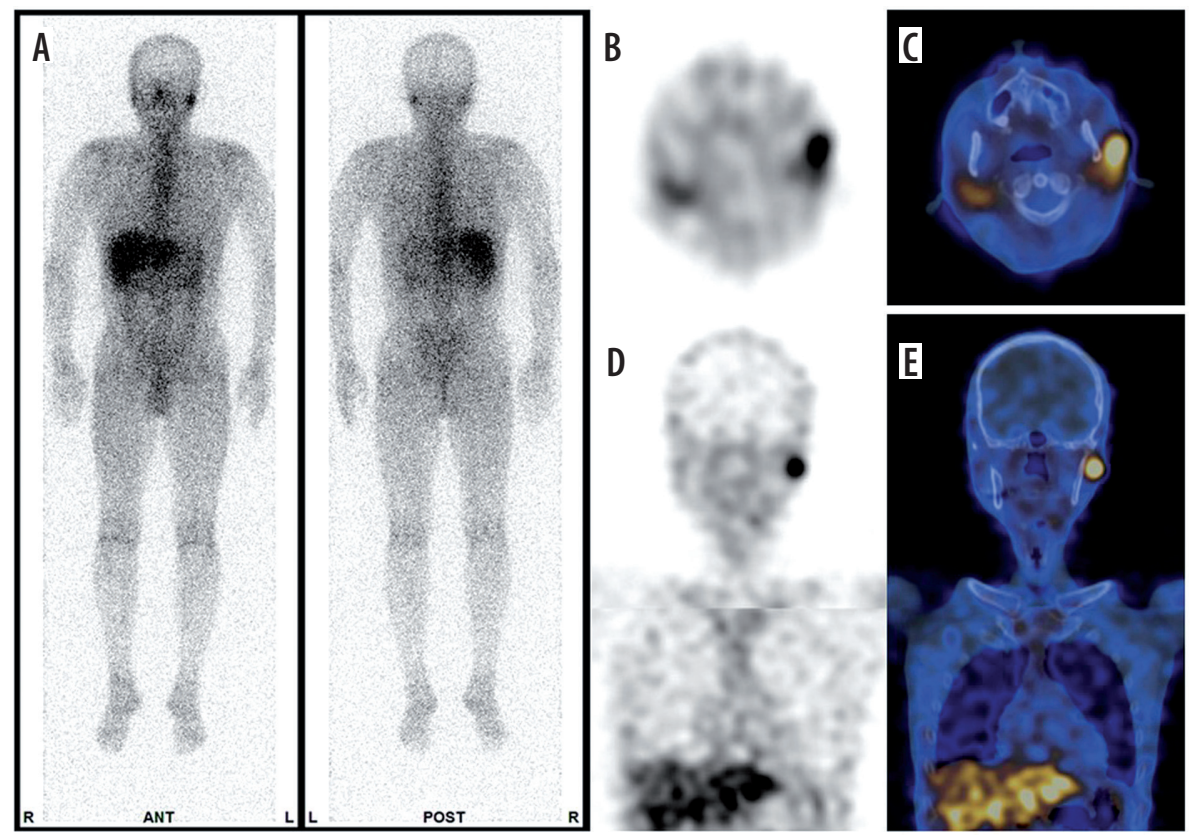

Figure 1. Malignant lymphoma of the left side of the neck in a 54-year-old male. A) planar, B) transaxial single-photon emission tomography (SPECT) and C) single-photon emission-computed tomography (SPECT-CT), D) coronal SPECT and E) SPECT-CT show increased uptake

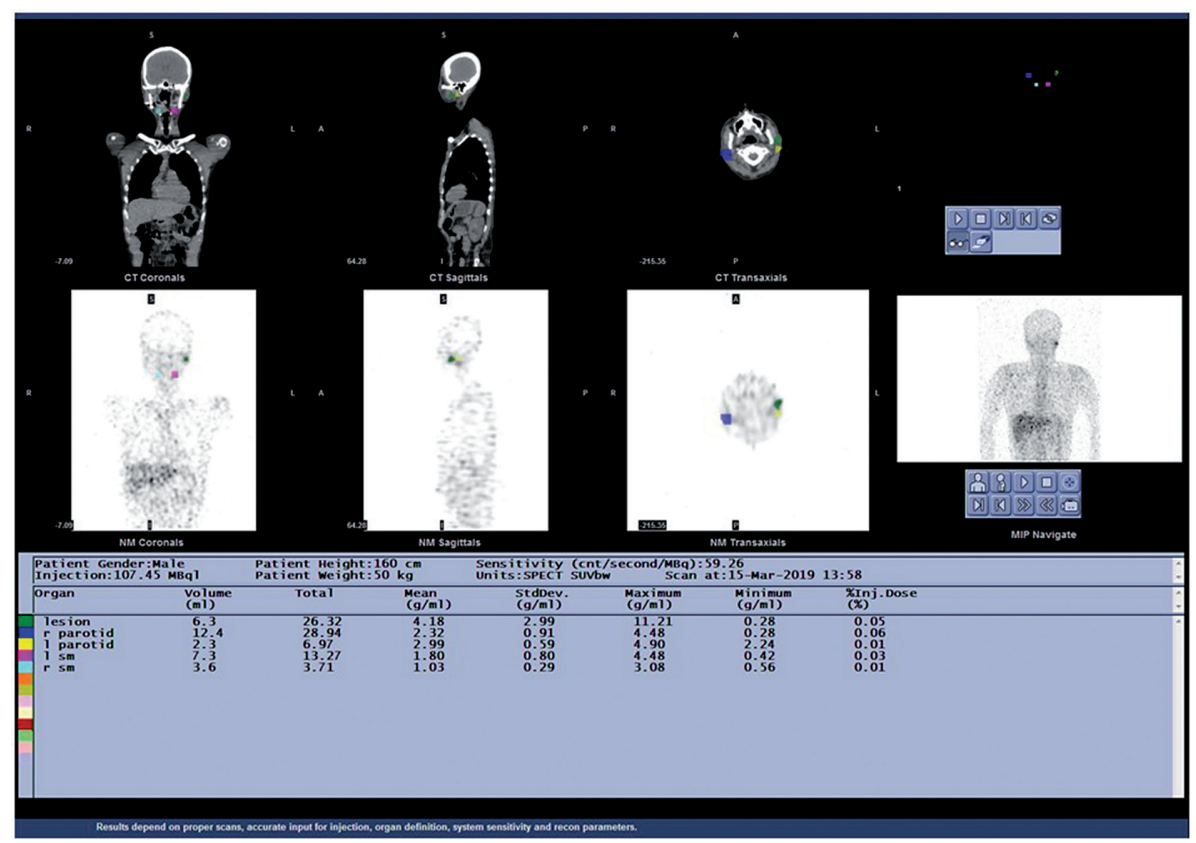

Figure 2. Malignant lymphoma of the left side of the neck in a 54-year-old male. By using the transaxial single-photon emission tomography (SPECT) and computed tomography (CT) as the anatomical reference, a volume of interest (VOI) was drawn over the lesion (green), right parotid gland (blue), left parotid gland (yellow), right submandibular gland (sky blue), and left submandibular gland (pink). The dosimetry software provided multiple quantitative data for a given VOI

carcinoma (two tongue and one mandible), three epithelial dysplasia (one tongue, one buccal mucosa, and one floor of the mouth), one squamous cell carcinoma (tongue), one osteoradionecrosis (mandible), and four postoperative follow-up of malignant tumours (palatal adenoid cystic carcinoma, palatal squamous cell carcinoma, mandibular squamous cell carcinoma, and maxillary malignant mela- noma). Regarding the mechanism of ${ }^{67} \mathrm{Ga}$ accumulation in tumours, Tsan et al. [19] showed that ${ }^{67} \mathrm{Ga}$ was delivered to the tumour through capillaries with increased permeability, and ${ }^{67} \mathrm{Ga}$ binding proteins might also contribute to the accumulation and retention of ${ }^{67} \mathrm{Ga}$ in tumours. We consider that the size of tumours also is a factor of the degree of ${ }^{67} \mathrm{Ga}$ accumulation in lesions. 

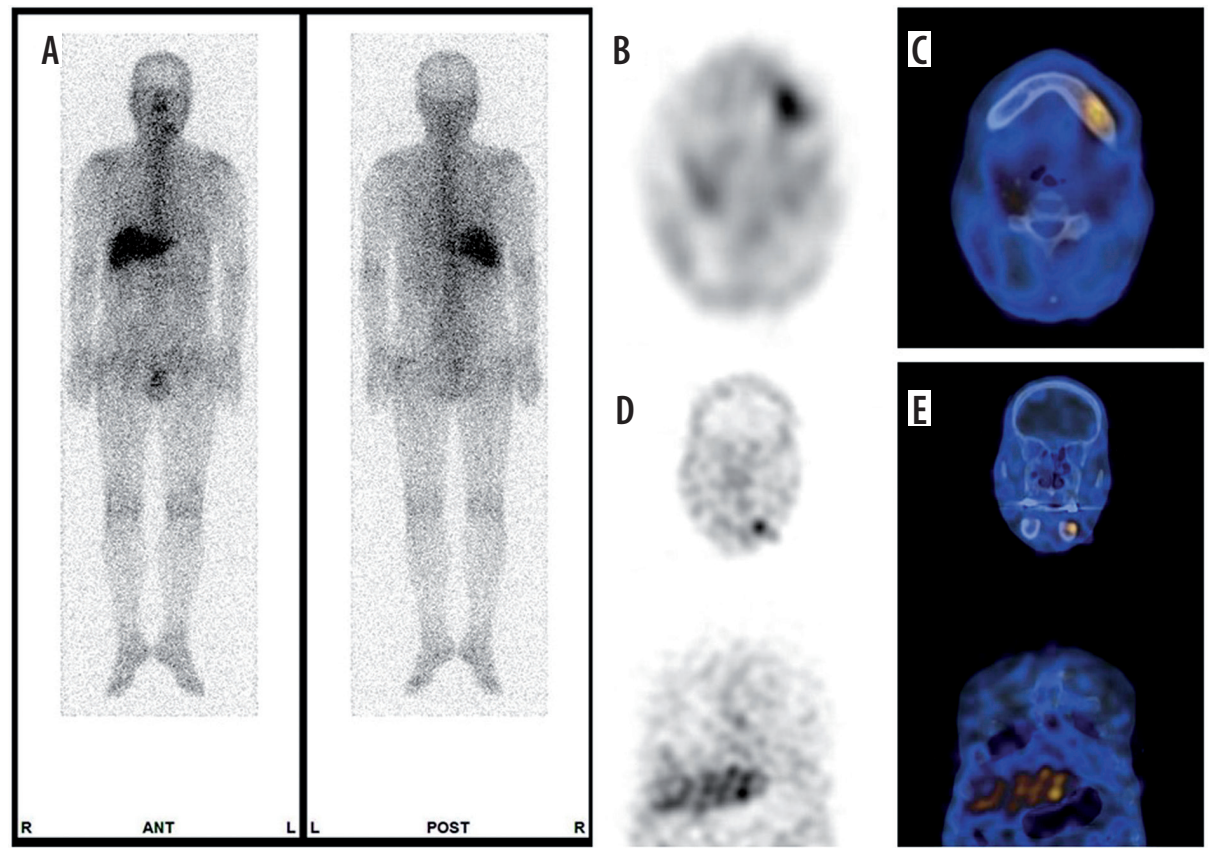

Figure 3. Osteomyelitis of the left side of the mandible in a 66-year-old male. A) planar, B) transaxial single-photon emission tomography (SPECT) and C) single-photon emission-computed tomography (SPECT-CT), D) coronal SPECT and E) SPECT-CT show increased uptake

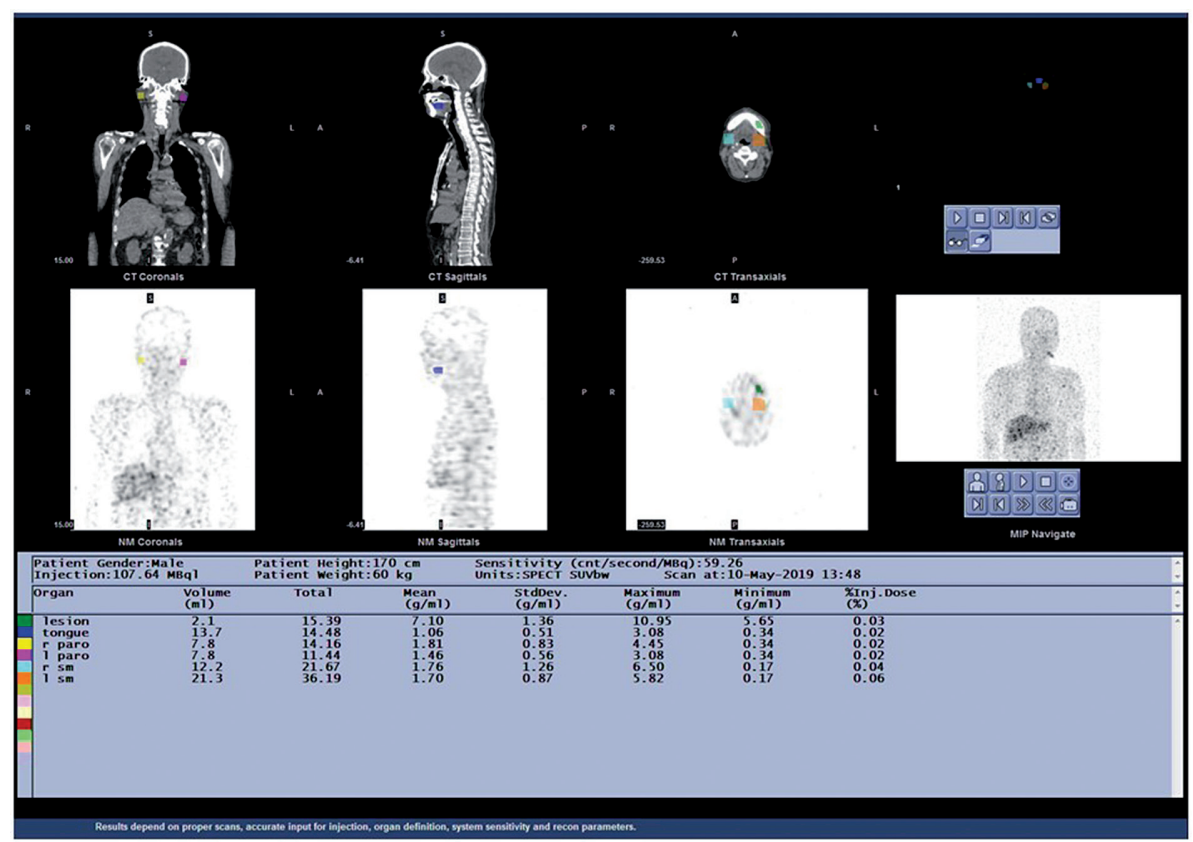

Figure 4. Osteomyelitis of the left side of the mandible in a 66-year-old male. By using the transaxial single-photon emission tomography (SPECT) and computed tomography (CT) as the anatomical reference, a volume of interest (VOI) was drawn over the lesion (green), right parotid gland (yellow), left parotid gland (pink), right submandibular gland (sky blue), and left submandibular gland (orange). The dosimetry software provided multiple quantitative data for a given VOI

Li et al. [1] indicated that ${ }^{67} \mathrm{Ga}$ scintigraphy for two of 11 patients who had chronic inflammatory lesions $(1 / 4$ parotitis, 1/5 submaxillaritis, and 0/2 lymphadenitis) were positive (18.2\%). Tsan et al. [19] showed that some in tumours may be taken up by inflammatory cells when they are present. Furthermore, Keijsers et al. [8] showed that imaging the inflammatory activity of sarcoidosis, namely the overall sensitivity, to detect active sarcoidosis was $88 \%$ for ${ }^{67} \mathrm{Ga}$ imaging. Ishii et al. [9] showed that ${ }^{67} \mathrm{Ga}$ scintigraphy was useful in differentiating between sarcoidosis and IgG4-related disease. Tsai et al. [12] suggested that the kidney uptake index from absolute quantitative renal ${ }^{67} \mathrm{Ga}$ scintigraphy may be a useful parameter for evaluating the disease activity in lupus nephritis. Ogura et al. 
[2-4] indicated that ${ }^{67} \mathrm{Ga}$ scintigraphy was an effective technique for detection of malignant tumours and inflammatory lesions of the head and neck. Szyszko et al. [20] showed the usefulness of PET-CT and PET-MRI in head and neck malignancy. However, to the best of our knowledge, ${ }^{67} \mathrm{Ga}$ SPECT-CT for the evaluation of head and neck has not been reported in the literature. Consequently, the authors consider that ${ }^{67} \mathrm{Ga}$ SPECT-CT is more useful for evaluation of malignant tumours and inflammatory diseases of the head and neck, although the successful clinical application of PET-CT and PET-MRI.

We have shown that $\mathrm{SUV}_{\max }$ for parotid glands and submandibular glands of all patients were $4.86 \pm 1.89$ and $4.64 \pm 1.64$, respectively. We consider that the evaluation of parotid glands and submandibular glands using ${ }^{67} \mathrm{Ga}$ SPECT-CT should be useful for diagnosis and treatment planning in head and neck clinical practice.

There were several limitations to this study. The sample was relatively small. Moreover, several types of tumours and inflammatory diseases of head and neck were studied. We consider that this study is a preliminary report, and further plans regarding greater number and types of pathology in subsequent works are necessary for differential diagnosis, and reassessment of treatment and prognostic factors.

\section{Conclusions}

This study investigated the ${ }^{67} \mathrm{Ga}$ SPECT-CT for the evaluation of head and neck, especially SUV $_{\max }$ of lesions, and the parotid and submandibular glands. ${ }^{67} \mathrm{Ga}$ SPECT-CT may be an effective technique for the evaluation of maxil-
Table 1. Gallium-67 SPECT-CT maximum SUV of parotid and submandibular glands

\begin{tabular}{|c|c|c|c|c|}
\hline \multirow[t]{2}{*}{ Characteristics } & \multirow{2}{*}{$\begin{array}{c}\text { Number of } \\
\text { salivary } \\
\text { glands }\end{array}$} & \multicolumn{2}{|c|}{ Maximum SUV } & \multirow[t]{2}{*}{$P$ value } \\
\hline & & Mean \pm SD & Range & \\
\hline \multicolumn{4}{|l|}{ Salivary glands } & \multirow[t]{3}{*}{0.694} \\
\hline Parotid gland & 28 & $4.86 \pm 1.89$ & $1.73-9.24$ & \\
\hline $\begin{array}{l}\text { Submandibular } \\
\text { gland }\end{array}$ & 28 & $4.64 \pm 1.64$ & $1.63-8.22$ & \\
\hline \multicolumn{4}{|l|}{ Age (years) } & \multirow[t]{3}{*}{0.544} \\
\hline$<70$ & 28 & $4.76 \pm 1.21$ & $3.05-7.85$ & \\
\hline$\geq 70$ & 28 & $4.74 \pm 2.20$ & $1.63-9.24$ & \\
\hline \multicolumn{4}{|l|}{ Gender } & \multirow[t]{3}{*}{0.320} \\
\hline Male & 24 & $4.87 \pm 1.24$ & $3.08-7.85$ & \\
\hline Female & 32 & $4.66 \pm 2.08$ & $1.63-9.24$ & \\
\hline Total & 56 & $4.75 \pm 1.76$ & $1.63-9.24$ & \\
\hline
\end{tabular}

SPECT - single-photon emission computed tomography, CT - computed tomography, SUV - standardised uptake value, SD - standard deviation.

lofacial lesions, although the successful clinical application of PET-CT and PET-MRI.

\section{Acknowledgments}

This work was supported by JSPS KAKENHI Grant Number JP 18K09754.

\section{Conflict of interest}

The authors declare that they have no conflict of interest.

\section{References}

1. Li N, Zhu W, Zuo S, et al. Value of gallium-67 scanning in differentiation of malignant tumors from benign tumors or inflammatory disease in the oral and maxillofacial region. Oral Surg Oral Med Oral Pathol Oral Radiol Endod 2003; 96: 361-367.

2. Ogura I, Oda T, Sue M, et al. Gallium-67 scintigraphy in differential diagnosis of malignant tumours from non-tumorous lesions of the maxilla. Chin J Dent Res 2017; 20: 219-223.

3. Ogura I, Sue M, Oda T, et al. Comparison between mandibular malignant tumors and inflammatory lesions using ${ }^{67} \mathrm{Ga}$ scintigraphy: relationship with panoramic radiography, CT and MRI findings. Int J Diagn Imaging 2017; 4: 67-73.

4. Ogura I, Oda T, Sue M, et al. Comparison between squamous cell carcinoma and inflammatory diseases of the oral and maxillofacial region using gallium-67 scintigraphy with computed tomography and magnetic resonance imaging. Pol J Radiol 2018; 83: e452-458.

5. Murata Y, Ishida R, Umehara I, et al. ${ }^{67} \mathrm{Ga}$ whole-body scintigraphy in the evaluation of head and neck squamous cell carcinoma. Nucl Med Commun 1999; 20: 599-607.

6. Kosuda S, Kadota Y, Umeda S, et al. Does supplementation of CT and MRI with gallium-67 SPECT improve the differentiation be- tween benign and malignant tumors of the head and neck? Ann Nucl Med 2003; 17: 475-480.

7. Okada M, Sato N, Ishii K, et al. FDG PET/CT versus CT, MR imaging, and $67 \mathrm{Ga}$ scintigraphy in the posttherapy evaluation of malignant lymphoma. Radiographics 2010; 30: 939-957.

8. Keijsers RG, Grutters JC, Thomeer M, et al. Imaging the inflammatory activity of sarcoidosis: sensitivity and inter observer agreement of $67 \mathrm{Ga}$ imaging and 18F-FDG PET. Q J Nucl Med Mol Imaging 2011; 55: 66-71.

9. Ishii S, Miyajima M, Sakuma K, et al. Comparison between sarcoidosis and IgG4-related disease by whole-body 67Ga scintigraphy. Nucl Med Commun 2013; 34:13-18.

10. Shim H, Joo J, Choi HJ, et al. Lack of increased FDG uptake in the lacrimal and salivary glands in patients with sarcoidosis and potential underlying mechanism. Clin Nucl Med 2016; 41: 274-277.

11. Aslangul E, M'bemba J, Caillat-Vigneron N, et al. Diagnosing diabetic foot osteomyelitis in patients without signs of soft tissue infection by coupling hybrid $67 \mathrm{Ga}$ SPECT/CT with bedside percutaneous bone puncture. Diabetes Care 2013; 36: 22032210 . 
12. Tsai SC, Hsieh TY, Huang PW, et al. Absolute quantitative evaluation of $67 \mathrm{Ga}$ scintigraphy in lupus nephritis. Clin Nucl Med 2016; 41: 442-446.

13. Suh MS, Lee WW, Km YK, et al. Maximum standardized uptake value of ${ }^{99 m}$ Tc hydroxymethylene diphosphonate SPECT/CT for the evaluation of temporomandibular joint disorder. Radiology 2016; 280: 890-896.

14. Kaneta T, Ogawa M, Daisaki H, et al. SUV measurement of normal vertebrae using SPECT/CT with Tc-99m methylene diphosphonate. Am J Nucl Med Mol Imaging 2016; 6: 262-268.

15. Kim J, Lee H, Lee $H$, et al. Quantitative single-photon emission computed tomography/computed tomography for evaluation of salivary gland dysfunction in Sjögren's syndrome patients. Nucl Med Mol Imaging 2018; 52: 368-376.

16. Ninomiya K, Toya S, Ogura I. Single-photon emission computed tomography/computed tomography for evaluation of salivary gland dysfunction: preliminary study on diagnostic ability of maximum standardized uptake value. Oral Radiol 2020; 36: 163-167.

17. Ogura I, Kobayashi E, Nakahara K, et al. Quantitative SPECT/CT imaging for medication-related osteonecrosis of the jaw: a preliminary study using volume-based parameters, comparison with chronic osteomyelitis. Ann Nucl Med 2019; 33: 776-782.

18. Ogura I, Sasaki Y, Sue M, et al. Tc-99m hydroxymethylene diphosphonate SPECT/CT for the evaluation of osteonecrosis of the jaw: preliminary study on diagnostic ability of maximum standardized uptake value. Clin Radiol 2020; 75: 46-50.

19. Tsan MF, Scheffel U. Mechanism of gallium-67 accumulation in tumors. J Nucl Med 1986; 27: 1215-1219.

20. Szyszko TA, Cook GJR. PET/CT and PET/MRI in head and neck malignancy. Clin Radiol 2018; 73: 60-69. 